multiple-locus variable-number tandem-repeat analysis to determine clonal spread of toxin A-negative Clostridium difficile in a general hospital in Buenos Aires, Argentina. Clin Microbiol Infect 2009;15:1080-1086.

3. van den Berg RJ, Claas EC, Oyib DH, et al. Characterization of toxin A-negative, toxin $\mathrm{B}$-positive Clostridium difficile isolates from outbreaks in different countries by amplified fragment length polymorphism and PCR ribotyping. J Clin Microbiol 2004; 42(3):1035-1041.

4. Gerding DN. Global epidemiology of Clostridium difficile infection in 2010. Infect Control Hosp Epidemiol 2010;31(suppl 1):S32S34.

5. Barbut F, Lalande V, Burghoffer B, Thien HV, Grimprel E, Petit JC. Prevalence and genetic characterization of toxin A variant strains of Clostridium difficile among adults and children with diarrhea in France. $J$ Clin Microbiol 2002;40:2079-2083.

6. Kato $\mathrm{H}$, Kato $\mathrm{N}$, Watanabe $\mathrm{K}$, et al. Identification of toxin Anegative, toxin B-positive Clostridium difficile by PCR. J Clin Microbiol 1998;36:2178-2182.

7. Geric B, Rupnik M, Gerding DN, Grabnar M, Johnson S. Distribution of Clostridium difficile variant toxinotypes and strains with binary toxin genes among clinical isolates in an American hospital. J Med Microbiol 2004;53(9):887-894.

8. Rupnik M, Kato N, Grabnar M, Kato $H$. New types of toxin Anegative, toxin $\mathrm{B}$-positive strains among Clostridium difficile isolates from Asia. J Clin Microbiol 2003;41(3):1118-1125.

9. Kato H, Kato N, Katow S, Maegawa T, Nakamura S, Lyerly DM. Deletions in the repeating sequences of the toxin A gene of toxin A-negative, toxin B-positive Clostridium difficile strains. FEMS Microbiol Lett 1999;175:197-203.

\section{Prevalence of Nasal Carriage of Mupirocin- Resistant Staphylococcus aureus among Hospitalized Patients in Thailand}

To the Editor-In various parts of the world, mupirocin has been used as a component of a "search and destroy" strategy to prevent methicillin-resistant Staphylococcus aureus (MRSA) infection prior to a surgical procedure. ${ }^{1-3}$ Increased use of mupirocin has been associated with an increased prevalence of mupirocin-resistant MRSA., ${ }^{4,5}$ In Thailand, there is neither routine surveillance for nasal carriage of MRSA nor attempts to eradicate carriage among hospitalized inpatients. Given that mupirocin and antibiotics can be purchased at outpatient pharmacies in Thailand without a prescription, ${ }^{6}$ it is expected that the prevalence of mupirocin-resistant MRSA nasal carriage is high. We performed a point prevalence study to evaluate the prevalence of MRSA nasal carriage and to estimate the proportion of individuals with mupirocin-resistant MRSA nasal carriage among admitted inpatients at Thammasat University Hospital.

From January 1 to January 7,2010, we cultured anterior nares swab specimens to detect MRSA carriage. Consecutive hospitalized patients who consented to the study participation on day 1 of hospitalization provided swab samples from both anterior nares that were then cultured for presence of MRSA. Information regarding demographics and clinical history of previous admission and exposure to antibiotics (in both inpatient and outpatient settings) was collected from each patient. Detection of MRSA from swab specimens was performed as previously described. ${ }^{4}$ Samples containing MRSA isolates from all patients who tested positive for MRSA carriage were then subcultured to $\mathrm{BBL}$ trypticase soy agar with $5 \%$ sheep blood (BD Diagnostics) and incubated at $35^{\circ} \mathrm{C}$ for 24 hours. Colonies isolated from the BBL trypticase soy agar were then inoculated on a Mueller Hinton II plate, and a mupirocin Etest ( $\mathrm{AB}$ Biodisk) strip was applied. After 24 hours of incubation at $35^{\circ} \mathrm{C}$, the minimum inhibitory concentration (MIC) was read. Isolates were classified as susceptible (MIC, $<8 \mu \mathrm{g} / \mathrm{mL}$ ), low-level resistant (MIC, 8-256 $\mu \mathrm{g} / \mathrm{mL}$ ), or high-level resistant (MIC, $512 \mu \mathrm{g} / \mathrm{mL}$ ).

During the study period, $250(86 \%)$ of 290 adult patients who were admitted to all units at Thammasat University Hospital provided consent and were enrolled in the study; 149 $(60 \%)$ were male, and the median age was 45 years (range, 20-89 years). Nasal carriage of MRSA was detected in 9 patients $(3.6 \%)$. Four $(44 \%)$ of these 9 patients were admitted to medical units, 3 (33\%) were admitted to surgical units, and $2(23 \%)$ were admitted to an orthopedic unit. All patients with nasal carriage of MRSA had a history of hospital admission within the past 12 months. Low-level mupirocinresistant MRSA was detected in 2 patients (22\%). These 2 patients had a history of skin abrasions and had self-purchased mupirocin and fluoroquinolones from a local pharmacy within 3 months of admission to the hospital.

Our data suggest that MRSA nasal carriage occurred among patients who had a history of contact with the Thai health care system, and that injudicious use of mupirocin in the community may be associated with carriage of low-level mupirocin-resistant MRSA isolates. The relatively low prevalence of MRSA nasal carriage (3.6\%) among hospitalized patients in our study suggests that a search and destroy strategy may not be cost-effective to implement in this middle-income country, while the relatively high prevalence of mupirocinresistant MRSA (22\%) among patients with nasal carriage of MRSA suggests the need to employ an antimicrobial stewardship program at the community level to help limit the unnecessary use of mupirocin and other antibiotics. Further studies are needed to evaluate the relationship between the unnecessary use of mupirocin in the community and the emergence of mupirocin-resistant MRSA among hospitalized inpatients and to evaluate the cost-effectiveness of a search and destroy strategy for MRSA in Thailand.

\section{ACKNOWLEDGMENTS}

Financial support. This study was supported by the National Research University Project of Thailand Office of Higher Education Commission (to A.A.). 
Potential conflicts of interest. All authors report no conflicts of interest relevant to this article.

\section{Anucha Apisarnthanarak, $M D_{;}^{1}$ David K. Warren, $\mathrm{MD}_{;}^{2}$ Victoria J. Fraser, $\mathbf{M D}^{2}$}

Affiliations: 1. Division of Infectious Diseases, Thammasat University Hospital, Pratumthani, Thailand; 2. Washington University School of Medicine, Saint Louis, Missouri.

Address correspondence to Anucha Apisarnthanarak, MD, Division of Infectious Diseases, Thammasat University Hospital, Pratumthani, Thailand, 12120 (anapisarn@yahoo.com).

Infect Control Hosp Epidemiol 2011;32(5):522-523

(C) 2011 by The Society for Healthcare Epidemiology of America. All rights reserved. 0899-823X/2011/3205-0022\$15.00. DOI: 10.1086/659953

\section{REFERENCES}

1. Perl TM, Cullen JJ, Wenzel RP, et al. Intranasal mupirocin to prevent postoperative Staphylococcus aureus infections. $N$ Engl J Med 2002;346:1871-1877.

2. Wilcox $\mathrm{MH}$, Hall J, Pike $\mathrm{H}$, et al. Use of perioperative mupirocin to prevent methicillin-resistant Staphylococcus aureus (MRSA) orthopaedic surgical site infections. J Hosp Infect 2003;54:196-201.

3. Jog $S$, Cunningham $R$, Cooper $S$, et al. Impact of preoperative screening for methicillin-resistant Staphylococcus aureus by realtime polymerase chain reaction in patients undergoing cardiac surgery. I Hosp Infect 2008;69:124-130.

4. Jones JC, Rogers TJ, Brookmeyer P, et al. Mupirocin resistance in patients colonized with methicillin-resistant Staphylococcus aureus in a surgical intensive care unit. Clin Infect Dis 2007;45:541547.

5. Cookson $\mathrm{BD}$. The emergence of mupirocin resistance: a challenge to infection control and antibiotic prescribing practice. $J$ Antimicrob Chemother 1998;41:11-18.

6. Apisarnthanarak A, Tunpornchai J, Tanawitt K, Mundy LM. Nonjudicious dispensing of antibiotics by drug stores in Pratumthani, Thailand. Infect Control Hosp Epidemiol 2008;29:572-575.

\section{Pseudo-outbreak of Pseudomonas putida Respiratory Infection Caused by Laboratory Contamination}

To the Editor-Pseudomonas putida is a gram-negative, aerobic bacterium that is a common inhabitant of soil, plants, and water. It has been found to cause septicemia in immunocompromised patients, ${ }^{1,2}$ and nosocomial transmission has been reported, associated with contaminated heparin or antiseptic solutions. ${ }^{3-5}$ Nevertheless, its isolation from clinical specimens is rare, and it is usually considered an environmental contaminant. $P$. putida has also been reported as a cause of pseudo-outbreaks in contaminated urine collection kits and contaminated commercial antifog solutions. ${ }^{6.7} \mathrm{We}$ now report a pseudo-outbreak of $P$. putida respiratory infections, involving 5 patients, caused by an automated spiral plater.
The infection control unit was notified on October 2, 2009, of a cluster of $P$. putida isolations from blind distal bronchial samples from 3 patients undergoing ventilation in the intensive care unit (ICU). The first positive sample had been recorded 5 days before, and the two others the day of notification. In each sample, $P$. putida was isolated among other bacteria at a significant level (at least $10^{3} \mathrm{cfu} / \mathrm{mL}$ ). Review of the previous 3 months of microbiology laboratory records found 2 other instances of $P$. putida isolation, one from a bronchial sample of another ICU patient and the other from sputum of a patient in the pulmonology department. All isolates presented an identical antibiotic susceptibility pattern. During the same period, $P$. putida was not isolated from other clinical specimens. A thorough ward-based investigation revealed no epidemiological link to suggest cross-infection between the patients. In particular, the pulmonology department patient had never been hospitalized in the ICU and did not share any device with the ICU patients. Therefore, the investigation focused on the microbiology laboratory, where the 5 samples were processed by the same device (a Whitley Automated Spiral Plater WASP 2; Don Whitley Scientific) dedicated to the clinical respiratory samples.

The WASP 2 was used for many years without any problem. It is a fully automated spiral plater, able to load a sample with a stylus, inoculate a plate, clean the stylus in a sanitizing solution $(70 \%$ alcohol), and finally rinse the stylus with sterile water loaded from a $110-\mathrm{mL}$ reusable container (Figure 1). The recommendation of the WASP 2 user manual ${ }^{8}$ is to sterilize the containers filled with sterile water by autoclaving.

Laboratory procedures for handling specimens and cleaning processes were reviewed with laboratory personnel. Aseptically collected samples of domestic water, demineralized rinse water, stylus, sanitizing solution, and each of the 12 reusable containers were obtained for bacterial culture. For the stylus, a $100-\mu \mathrm{L}$ aliquot of sterile water was loaded by the stylus and directly deposited on a plate. For domestic water, demineralized water, sanitizing solution, and reusable containers containing $110 \mathrm{~mL}$ of sterile water, $100 \mathrm{~mL}$ of liquid were filtered and inoculated on plates. Cultures of all specimens were obtained using conventional microbiologic methods. Restriction endonuclease DNA profiles were determined by pulsed-field gel electrophoresis for all available isolates, using the restriction enzyme SpeI.

There was no change in personnel, microbiological technique, or culture medium. The review of the procedures with the microbiology laboratory personnel revealed a change in the process of reusable-container disinfection a few weeks before the first case occurred. The autoclave usually used to sterilize the containers filled with sterile water had broken down, and an alternate procedure consisting of a chemical disinfection was performed until the autoclave was repaired. However, as the written procedure requested a rinse with sterile water after immersion in a bactericidal solution (DDN 250, Franklab Laboratory) for 60 minutes, the employee responsible for container disinfection had immersed the con- 